\title{
641.
}

\section{ON THE FLEXURE OF A SPHERICAL SURFACE.}

[From the Messenger of Mathematics, vol. vi. (1877), pp. 88-90.]

IT is known that an inextensible spherical surface, or to fix the ideas the spherical quadrilateral included between two arcs of meridian and two arcs of parallel, may be bent in suchwise as to be part of a surface of revolution, the meridians and parallels of the spherical surface being meridians and parallels of the new surface, and, moreover, the radius of each parallel of the spherical surface being in the new surface altered in the constant ratio $k$ to 1 . We have, in fact, on the spherical surface, writing $p$ for the latitude and $q$ for the longitude, and the radius being unity,

values which give

$$
\begin{aligned}
& x=\cos p \cos q \\
& y=\cos p \sin q, \\
& z=\sin p
\end{aligned}
$$

$$
d x^{2}+d y^{2}+d z^{2}=d p^{2}+\cos ^{2} p d q^{2}
$$

This last equation is satisfied by the values

$$
\begin{aligned}
& x=\cos p \cos \frac{q}{k}, \\
& y=\cos p \sin \frac{q}{k}, \\
& z=E(k, p),
\end{aligned}
$$

where $E(k, p),=\int_{0} \sqrt{ }\left(1-k^{2} \sin ^{2} p\right) d p$, is the elliptic function of the second kind; or rather, this is so when $k<1$, but the same notation may be used when $k>1$. These values give the deformation in question.

The two cases to be considered are $k<1$, and $k>1$; we take in each case a spherical quadrilateral $A B C D$ (fig. 1), bounded by $A B$ (an arc of the equator), the arc of parallel $C D$, and the two arcs of meridian $A D$ and $B C$. In the first case, there is no limit to the latitude $A D,=B C$, or taking these $=90^{\circ}$, we may in place 
of the quadrilateral $A B C D$ consider the birectangular triangle $A B E$; the new form of this is $A^{\prime} B^{\prime} E^{\prime}$, where the radius $O A^{\prime},=k \cdot O A,=k$, is less than the original radius

Fig. 1.

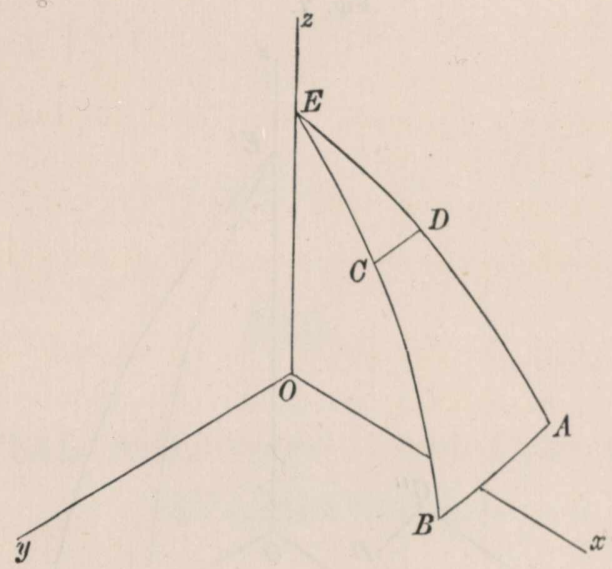

unity, but $O E^{\prime},=E_{1} k$, is greater than the same radius unity. The surface has at $E^{\prime}$ a conical point, the semi-aperture of the cone being $=\tan ^{-1} \frac{1}{k^{\prime}}$, if as usual $k^{\prime}=\sqrt{ }\left(1-k^{2}\right)$; to verify this, writing for convenience $q=0$, we have for the meridian section $x=\cos p$, $z=E(k, p)$, and thence $\frac{d x}{d z}=-\frac{\sqrt{ }\left(1-k^{2} \sin ^{2} p\right)}{\sin p}$, which for $p=90^{\circ}$ gives $\frac{d z}{d x}=-k^{\prime}$. Observe also that for $p=0, \frac{d z}{d x}=\infty$, viz. the surface of revolution cuts the plane of the equator at right angles.

There is no limit to the arc $A B$, it may be $=360^{\circ}$, viz. we must in this case cut the hemisphere along a meridian to allow of the deformation; or it may exceed $360^{\circ}$, the hemisphere spherical surface being in this case conceived of as wrapping indefinitely over itself, and we may instead of the half lune $E^{\prime} A^{\prime} B^{\prime}$, consider the lune included between two meridians extending from pole to pole, and therefore the whole spherical surface, conceived of as wrapping indefinitely over itself; the result is, that this may be deformed into a surface of revolution, which, in its general form, resembles that obtained by the revolution of an are less than a semi-circle round its chord; the half-chord being greater, and the versed-sine less than the radius of the original sphere.

If $k>1$, there is obviously a limit to the latitude $A D,=B C$, of the spherical quadrilateral; viz. this is equal to $\sin ^{-1} \frac{1}{k}$. Supposing that in the quadrilateral $A B C D$ (fig. 1) the latitude has this limiting value, then (see fig. 2) the new form is $A^{\prime \prime} B^{\prime \prime} C^{\prime \prime} D^{\prime \prime}$, where along the bounding arc $C^{\prime \prime} D^{\prime \prime}$ the tangent plane is horizontal; viz. as before $\frac{d z}{d x}=-\frac{\sqrt{ }\left(1-k^{2} \sin ^{2} p\right)}{\sin p},=0$ for $p=\sin ^{-1} \frac{1}{k}$. It is to be observed, that the radii for the parallels $A^{\prime \prime} B^{\prime \prime}$ and $C^{\prime \prime} D^{\prime \prime}$ are $k$ and $k \cos p$ respectively; the difference of 
these is $k(1-\cos p)$, which, however great $k$ is, must be less than the arc of meridian $A^{\prime \prime} D^{\prime \prime},=p ;$ substituting for $k$ the value $\frac{1}{\sin p}$, the condition is $\frac{1-\cos p}{\sin p}<p$, viz. this

Fig. 2.

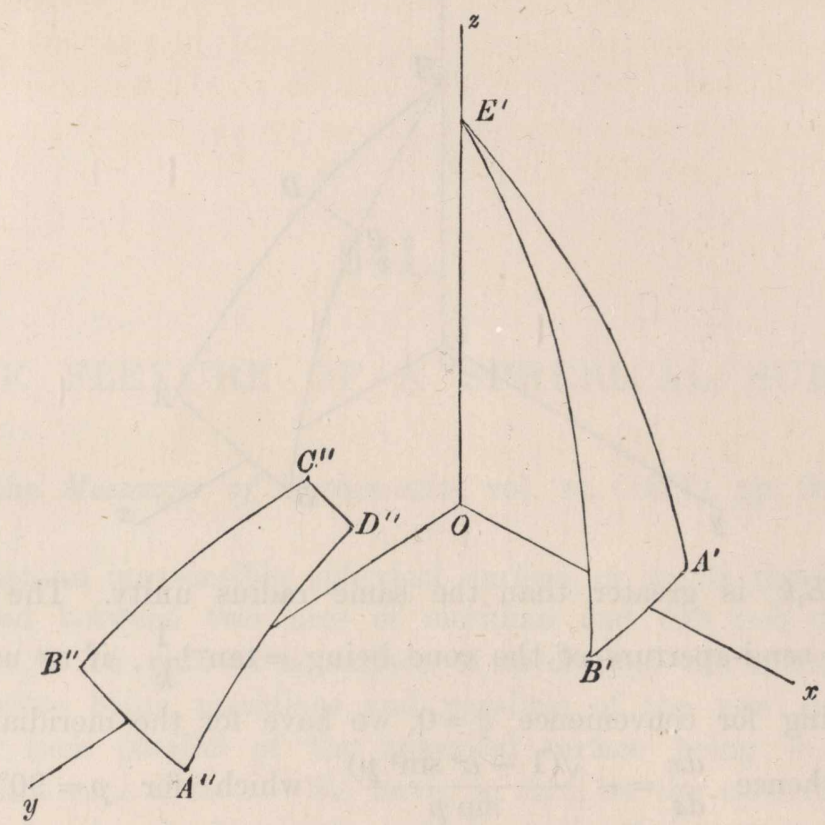

is $\tan \frac{1}{2} p<p$, which is true for every value up to $p=90^{\circ}$. But, more than this, we should have

$$
k^{2}(1-\cos p)^{2}+E^{2}(k, p)<p^{2},
$$

viz. writing as before $k=\frac{1}{\sin p}$, this is

$$
E^{2}\left(\frac{1}{\sin p}, p\right)<p^{2}-\tan ^{2} \frac{1}{2} p
$$

this must be true, although (relating as it does to a form of $E$ for which $k$ is greater than 1) there might be some difficulty in verifying it.

There is, as in the first case, no limit to the value of $A B$, viz. this may be $=360^{\circ}$, the spherical zone being then cut along a meridian, or it may be greater than $360^{\circ}$; and, moreover, the spherical quadrilateral may extend south of the equator, but of course so that the limiting south latitude does not extend beyond the foregoing value $\sin ^{-1} \frac{1}{k}$ : viz. we may have a zone between the latitudes $\pm \sin ^{-1} \frac{1}{k}$, which may be a complete zone from longitude $0^{\circ}$ to $360^{\circ}$ or to any greater value than $360^{\circ}$. The result is, that the zone is deformed into a surface of revolution, which in its general form resembles that obtained by the revolution of a half-circle or half-ellipse about a line parallel to and beyond its bounding diameter, the bounding half-diameter being less, and the greatest radius of rotation greater, than the radius of the original sphere. 